\title{
Properties of exact and approximate traveling wave solutions for transport with nonlinear and nonequilibrium sorption
}

\author{
Werner Simon and Peter Reichert \\ Swiss Federal Institute for Environmental Science and Technology (EAWAG), Dübendorf, Switzerland \\ Christoph Hinz \\ Institute of Terrestrial Ecology, Swiss Federal Institute of Technology (ETH), Schlieren, Switzerland
}

\begin{abstract}
Nonlinear sorption leads to the existence of moving concentration fronts of substances transported in porous media that do not change shape. The cause for the existence of such traveling wave fronts is a balance between the self-sharpening effect of nonlinear sorption and the spreading effects of dispersion and sorption kinetics. While analytical solutions for the transport of nonlinearly sorbing substances are usually not available, the asymptotic front speed and the shape of traveling waves can in some situations be determined analytically. This is done by deriving a traveling wave equation in a moving coordinate system. In the case of simultaneous linear equilibrium and nonlinear nonequilibrium sorption, this traveling wave equation is a second-order differential equation. Because this equation cannot be solved analytically for typical nonlinear sorption isotherms, the second-order term is usually neglected. In this paper the significance of the second-order term is discussed for a simple piecewise linear sorption isotherm that allows the analytical solution of both the full and the simplified differential equations. These analytical solutions make it possible to calculate the approximation error as a function of flow and isotherm parameters and thus to localize domains where the error may be significant. In addition, the analytical solutions are used to discuss the identifiability of model parameters from asymptotic front shape data.
\end{abstract}

\section{Introduction}

In recent years pollution of groundwater aquifers has become an important issue. In order to assess the subsurface water quality, information on the mobility of pollutants, such as organic compounds and heavy metals, is required.

An important factor influencing the mobility of a compound is its sorption behavior to the surfaces present in the aquifer. The sorption equilibrium is usually characterized by the sorption isotherm, which can be a linear or a nonlinear function of the dissolved concentration. If the sorption process is slow (due to the kinetics of the chemical processes or due to diffusive mass transfer limitations), information on sorption kinetics is necessary in addition to the knowledge of the shape of the isotherm in order to correctly describe the transport processes.

Unfortunately, the advection-dispersion equation with nonlinear sorption cannot be solved analytically. The effect of nonlinear sorption is to make the effective velocity of a concentration wave transported with water flow in a porous medium dependent on the concentration. This leads to a selfsharpening effect at the front of a concentration pulse and a spreading effect on its tail or vice versa. In the absence of dispersion and kinetic sorption effects, this leads to a discontinuous "shock wave" solution [Helfferich and Klein, 1970; Charbeneau, 1981; Bolt, 1982; Rhee et al., 1989; Schweich et al., $1993 a, b]$. From a mathematical point of view this is the same phenomenon as the formation of shock waves in the kinematic approximation to fluid flow [Whitham, 1974; Lighthill, 1978;

Copyright 1997 by the American Geophysical Union.

Paper number 97WR00301.

0043-1397/97/97WR-00301\$09.00
Smoller, 1983]. If dispersion and/or sorption kinetics are present, the self-sharpening effect of nonlinear sorption on a concentration front finally becomes balanced by the dispersive effect of these processes and a continuous shock layer develops. The asymptotic form of such a shock layer can be calculated by searching for so-called traveling wave solutions that are steady state solutions in a coordinate system moving at a constant velocity. In contrast to the full partial differential equation, in some cases there exist analytical solutions to the ordinary differential equation describing traveling waves also in the presence of nonlinear sorption. The existence of such analytical solutions very much simplifies the discussion of the effect of model parameters on measurable concentration profiles and, conversely, the discussion of the identifiability of processes from measurements.

Traveling wave solutions for the transport of sorbing substances in porous media have been investigated under different assumptions. First, traveling wave solutions for dispersive transport with nonlinear equilibrium sorption were studied [Rhee et al., 1971]. This result was subsequently extended to the case of dispersive transport with nonlinear nonequilibrium sorption [Rhee and Amundson, 1972, 1974; van Duijn and Knabner, 1992a, b]. A further extension to the case of dispersive transport with linear equilibrium and nonlinear nonequilibrium sorption has been studied by van der Zee [1990]. Furthermore, a traveling wave solution for first-order decay and nonlinear sorption has been derived [Bosma and van der Zee, 1993].

The partial differential equation describing transport under all of the above mentioned conditions simplifies to a first- or second-order ordinary differential equation for traveling wave 
solutions. The second-order term in this equation appears only if dispersion and kinetic sorption effects are simultaneously present. It is usually neglected because in most cases of practical relevance it is much smaller than the other terms and its neglection makes it possible to solve the equation analytically for typical nonlinear sorption isotherms [Rhee and Amundson, 1972; van der Zee, 1990]. However, we know of only one numerical study that justifies this procedure [Rhee and Amundson, 1972]. The numerical solution of the traveling wave equation is not trivial because the integration domain of the differential equation extends to infinity and the boundary conditions must therefore be fulfilled asymptotically.

It is the goal of this paper to discuss the importance of the second-order term for a special piecewise linear sorption isotherm that allows the analytical solution of both the complete and the simplified traveling wave equation. These results are complementary to the numerical results cited above; they can be applied only to a limited class of isotherms, but they make it possible to discuss the behavior of the solutions much more easily than with the numerical procedure. The consequences of the dependence of the shape of the traveling wave front on the model parameters for the identification of dispersion and sorption processes are discussed.

\section{Theory}

\subsection{Brief Review of Previous Work}

Because of the strong connection of this paper to the work by van der Zee, we use the notation of one of his papers [van der Zee, 1990]. The two site model with linear equilibrium and nonlinear nonequilibrium sites can be written in the following form [van der Zee, 1990, equation (9)]:

$$
\begin{gathered}
\theta \frac{\partial c}{\partial t}+\frac{\partial q_{1}}{\partial t}=\theta D \frac{\partial^{2} c}{\partial z^{2}}-\nu \theta \frac{\partial c}{\partial z}-\frac{\partial q_{2}}{\partial t} \\
q_{1}=k_{l} c \\
\frac{\partial q_{2}}{\partial t}=k_{r}\left[f(c)-q_{2}\right]
\end{gathered}
$$

where $t$ is time $(T), z$ is the space coordinate in flow direction $(L), c$ is the dissolved substance concentration $\left(M L^{-3}\right), q_{1}$ is the water-equivalent concentration of substance sorbed to the linear equilibrium sites of type $1\left(M L^{-3}\right), q_{2}$ is the waterequivalent concentration of substance sorbed to the nonlinear nonequilibrium sites of type $2\left(M L^{-3}\right), \theta$ is the volumetric water content $(-), D$ is the dispersion coefficient $\left(L^{2} T^{-1}\right), \nu$ is the pore water velocity $\left(L T^{-1}\right), k_{l}$ is the linear sorption coefficient for sorption to the linear equilibrium sites $1(-)$, $k_{r}$ is the first-order rate coefficient for sorption to the nonequilibrium sites $2\left(T^{-1}\right)$, and $f(c)$ is the nonlinear sorption isotherm $\left(M L^{-3}\right)$. In (1) the adsorbed substance is expressed on a volumetric basis, that is,

$$
q_{\imath}=\rho s_{\imath}
$$

where $\rho$ is the bulk density of the solid phase $\left(M L^{-3}\right)$ and $s$, is the sorbed substance per unit solid phase mass $\left(M M^{-1}\right)$.

The following nondimensional parameters are introduced:

$$
R_{l}=1+\frac{k_{l}}{\theta}
$$

$$
\begin{gathered}
Z=\frac{z}{l} \\
T=\frac{\nu t}{R_{l} l} \\
P e=\frac{\nu l}{D} \\
L=\frac{k_{r} l R_{l}}{\nu} \\
W=\frac{1}{\theta R_{l}}
\end{gathered}
$$

where $R_{l}$ is the retardation factor for linear sorption, $l$ is the column length $(L), Z$ is the dimensionless spatial variable, $T$ is dimensionless time (or pore volume), $L$ is the dimensionless rate constant for sorption kinetics (Stanton number), and $P e$ is the Peclet number. Using these parameters, (1) changes to the nondimensional form

$$
\begin{gathered}
\frac{\partial c}{\partial T}=\frac{1}{P e} \frac{\partial^{2} c}{\partial Z^{2}}-\frac{\partial c}{\partial Z}-W \frac{\partial q_{2}}{\partial T} \\
\frac{\partial q_{2}}{\partial T}=L\left[f(c)-q_{2}\right]
\end{gathered}
$$

Van der Zee obtained the following differential equation for traveling wave solutions [van der Zee, 1990, equation (29)] to (4):

$$
\begin{aligned}
\left(\frac{\alpha}{P e L}\right) & \frac{d^{2} c}{d \eta^{2}}-\left(\frac{1}{P e}+\frac{\alpha(1-\alpha)}{L}\right) \frac{d c}{d \eta} \\
= & \alpha W\left(\frac{f\left(c_{0}\right)-f\left(c_{\imath}\right)}{c_{0}-c_{\imath}}\left(c_{0}-c\right)-\left[f\left(c_{0}\right)-f(c)\right]\right)
\end{aligned}
$$

where $\alpha$ is the nondimensional wave speed given by

$$
\alpha=\frac{1}{1+W \frac{f\left(c_{0}\right)-f\left(c_{\imath}\right)}{c_{0}-c_{\imath}}}
$$

$\eta$ is the nondimensional moving coordinate defined as

$$
\eta=Z-\alpha T
$$

and $c_{\imath}$ and $c_{0}$ are the initial concentration in the column and the step input concentration, respectively. Note that the wave speed $\alpha$ is independent of the parameters $P e$ and $L$. Equations (5) and (6) were already derived by Rhee and Amundson [1972] and Rhee et al. [1989], with the difference that these authors did not consider linear equilibrium sorption (in the present notation this corresponds to $k_{l}=0$ or $R_{f}=1$ ). Note that the second-order term in (5) is only present if both dispersion $(P e<\infty)$ and nonequilibrium sorption $(L<\infty)$ are present simultaneously. The wave speed is independent of dispersion and kinetic sorption effects; it is the same as the shock speed for nonlinear equilibrium sorption without dispersion. Dispersion and nonequilibrium sorption have exactly the same effect on the shape of the traveling wave if they do not occur simultaneously $(P e$ can be replaced by $L /[\alpha(1-\alpha)])$. Equation (5) must be solved for the boundary conditions

$$
c(-\infty)=c_{0} \quad c(+\infty)=c_{1}
$$


where $c_{t}$ is the initial concentration and $c_{0}$ the step concentration. Van der Zee solved (5) analytically for Langmuir and Freundlich adsorption isotherms neglecting the second-order term that makes analytical solution for these isotherms impossible [van der Zee, 1990].

If the nonlinear sorption isotherm $f(c)$ is twice differentiable, solutions to (5) exist only if one of the following conditions, (9a) or (9b), is satisfied [van der Zee, 1990, equation (20)]:

Convex isotherm:

$$
c_{0}>c_{\imath} \quad \frac{d f(c)}{d c}>0 \quad \frac{d^{2} f(c)}{d c^{2}}<0
$$

Concave isotherm:

$$
c_{0}<c_{\imath} \quad \frac{d f(c)}{d c}>0 \quad \frac{d^{2} f(c)}{d c^{2}}>0
$$

The convex case leads to a self-sharpening adsorption front, and the concave case to a self-sharpening desorption front. In this paper we concentrate on the convex case.

After solving (5), the concentrations at the linear equilibrium sites $q_{1}$ are given by

$$
q_{1}=k_{i} c
$$

(compare equation (1)), and those at the nonlinear nonequilibrium sites $q_{2}$ by the solution to the equation

$$
\frac{d q_{2}}{d \eta}=-\frac{L}{\alpha}\left[f(c)-q_{2}\right]
$$

with the boundary conditions

$$
\frac{d q_{2}}{d \eta}(-\infty)=0 \quad \frac{d q_{2}}{d \eta}(+\infty)=0
$$

[Van der Zee, 1990, equations (24b) and (25)].

\subsection{Solution for a Simple Piecewise Linear Isotherm}

2.2.1. Isotherm. In order to discuss the significance of the second-order term in (5) we choose the simplest possible nonlinear function $f(c)$ that leads to self-sharpening adsorption fronts (see discussion following (9)). This function shown in Figure 1 is given by

$$
\begin{gathered}
f(c)=\frac{f_{b}}{c_{b}} c \quad c \leq c_{b} \\
f(c)=f_{b}+a\left(c-c_{b}\right) \quad c \geq c_{b}
\end{gathered}
$$

with

$$
0 \leq a<\frac{f_{b}}{c_{b}}
$$

to guarantee convexity. In these equations, $c_{b}$ is the concentration at which the slope of the isotherm changes, $f_{b}$ is the corresponding value of the isotherm, and $a$ is the slope of the isotherm for concentrations larger than $c_{b}$ (Figure 1). This piecewise linear isotherm does not satisfy condition (9a) because it is not differentiable at the break point. Nevertheless, because of its convexity a traveling wave solution for an adsorption front exists.

For $a=0$ the isotherm given by (13) can be taken as a crude approximation to a Langmuir isotherm, for $0<a<f_{b} / c_{b}$ as an approximation to a Freundlich isotherm with an exponent

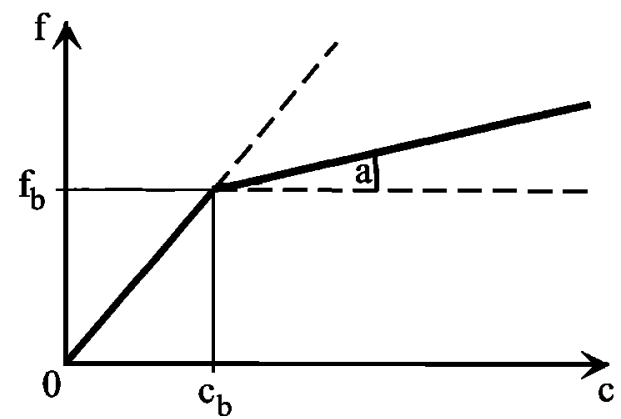

Figure 1. Plot of the piecewise linear, convex isotherm used in the present analysis.

smaller than 1 (a Freundlich isotherm with an exponent larger than 1 is concave, so that a self-sharpening desorption front appears instead of an adsorption front).

In order to obtain the self-sharpening effect for a step input, the concentration $c_{b}$ at which the slope of the isotherm changes must satisfy

$$
c_{\imath}<c_{b}<c_{0}
$$

This guarantees the behavior characteristic for a nonlinear convex isotherm.

2.2.2. Traveling wave equation. For the isotherm specified by (13) the differential equation (5) for $c$ simplifies to

$$
\begin{aligned}
\left(\frac{\alpha}{P e L}\right) & \frac{d^{2} c}{d \eta^{2}}-\left(\frac{1}{P e}+\frac{\alpha(1-\alpha)}{L}\right) \frac{d c}{d \eta} \\
= & \alpha W\left(\frac{f_{b}}{c_{b}}-a\right) \frac{c_{0}-c_{b}}{c_{0}-c_{l}}\left(c-c_{b}\right) \quad c \leq c_{b} \\
\left(\frac{\alpha}{P e L}\right) & \frac{d^{2} c}{d \eta^{2}}-\left(\frac{1}{P e}+\frac{\alpha(1-\alpha)}{L}\right) \frac{d c}{d \eta} \\
= & \alpha W\left(\frac{f_{b}}{c_{b}}-a\right) \frac{c_{b}-c_{i}}{c_{0}-c_{t}}\left(c_{0}-c\right) \quad c \geq c_{b}
\end{aligned}
$$

with

$$
\alpha=1 /\left(1+W \frac{\left(f_{b} / c_{b}\right)\left(c_{b}-c_{t}\right)+a\left(c_{0}-c_{b}\right)}{c_{0}-c_{\iota}}\right)
$$

This equation can be solved analytically with and without the second-order term $\alpha /(P e L) d^{2} c / d \eta^{2}$. A comparison of these two solutions allows us to discuss the significance of the second-order term.

2.2.3. Approximate analytical solution. The solution to (16) for the boundary conditions given by (8) with neglection of the second-order term $\alpha /(P e L) d^{2} c / d \eta^{2}$ is given by

$$
\begin{gathered}
c_{a p p}(\eta)=c_{0}-\left(c_{0}-c_{b}\right) \exp \left[\beta\left(\frac{f_{b}}{c_{b}}-a\right) \frac{c_{b}-c_{t}}{c_{0}-c_{\imath}}\left(\eta-\eta_{0}\right)\right] \\
\eta \leq \eta_{0}
\end{gathered}
$$

$$
c_{a p p}(\eta)=c_{\imath}+\left(c_{b}-c_{\imath}\right) \exp \left[-\beta\left(\frac{f_{b}}{c_{b}}-a\right) \frac{c_{0}-c_{b}}{c_{0}-c_{\imath}}\left(\eta-\eta_{0}\right)\right]
$$$$
\text { where }
$$ 


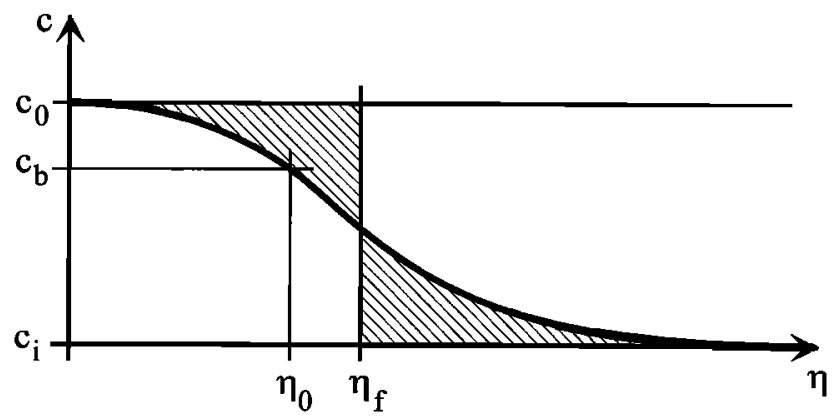

Figure 2. The front position $\eta_{f}$ is defined by the condition that the two hatched areas have the same size.

$$
\beta=\alpha W /\left(\frac{1}{P e}+\frac{\alpha(1-\alpha)}{L}\right)
$$

and $\eta_{0}$ is an integration constant describing the position where the solution $c_{a p p}$ is equal to $c_{b}$.

2.2.4. Exact analytical solution. The solution to the full equation (16) for the boundary conditions given by (8) is given by $c_{e x}(\eta)=c_{0}$

$$
\begin{aligned}
& -\frac{\left(c_{0}-c_{b}\right) \sqrt{1-X}+\left(c_{b}-c_{b}\right) \sqrt{1+Y}-\left(c_{0}-c_{b}\right)}{2 \sqrt{1-X}} \\
& \cdot \exp \left(\frac{\gamma}{2}(1+\sqrt{1-X})\left(\eta-\eta_{0}\right)\right) \\
& -\frac{\left(c_{0}-c_{b}\right) \sqrt{1-X}-\left(c_{b}-c_{b}\right) \sqrt{1+Y}+\left(c_{0}-c_{t}\right)}{2 \sqrt{1-X}} \\
& \cdot \exp \left(\frac{\gamma}{2}(1-\sqrt{1-X})\left(\eta-\eta_{0}\right)\right) \quad \eta \leq \eta_{0}
\end{aligned}
$$

$c_{e x}(\eta)=c_{\imath}+\left(c_{b}-c_{\imath}\right) \exp \left(-\frac{\gamma}{2}(\sqrt{1+Y}-1)\left(\eta-\eta_{0}\right)\right)$

$$
\eta \geq \eta_{0}
$$

with

$$
\begin{gathered}
\gamma=\frac{L}{\alpha}+\operatorname{Pe}(1-\alpha) \\
X=\frac{4 W P e L}{\gamma^{2}}\left(\frac{f_{b}}{c_{b}}-a\right) \frac{c_{b}-c_{t}}{c_{0}-c_{t}}
\end{gathered}
$$

and

$$
Y=\frac{4 W P e L}{\gamma^{2}}\left(\frac{f_{b}}{c_{b}}-a\right) \frac{c_{0}-c_{b}}{c_{0}-c_{1}}
$$

and where again $\eta_{0}$ is an integration constant describing the position where the solution $c_{e x}$ is equal to $c_{b}$. In order for the solution given above to be correct, it must be shown that $X$ is smaller than 1 . This can best be done by eliminating $W$ in (22) by using (17). This leads to the following form of $X$ :

$$
\begin{aligned}
X= & \frac{4}{\left(\left(\frac{P e}{L} \alpha(1-\alpha)\right)^{1 / 2}+\left[1 /\left(\frac{P e}{L} \alpha(1-\alpha)\right)^{1 / 2}\right]\right)^{2}} \\
& \cdot \frac{\frac{f_{b}}{c_{b}}\left(c_{b}-c_{\imath}\right)-a\left(c_{b}-c_{\imath}\right)}{\frac{f_{b}}{c_{b}}\left(c_{b}-c_{\imath}\right)+a\left(c_{0}-c_{b}\right)}
\end{aligned}
$$

Both factors in this representation of $X$ are smaller than or equal to 1 . Therefore this is also true for $X$ (very special parameter values would have to be chosen that $X$ would become equal to 1 ; therefore in all realistic situations $X$ will be smaller than 1 ).

2.2.5. Front position. In order to compare the approximate and exact solutions $c_{a p p}$ (18) and $c_{e x}$ (20) for the traveling wave front, the same front positions should be used. As shown in Figure 2, the front position $\eta_{f}$ can be defined by the condition that the integral from $-\infty$ to $\eta_{f}$ of the deviation of the concentration $c$ from the step input concentration $c_{0}$ is equal to the integral from $\eta_{f}$ to $\infty$ of the deviation of $c$ from the initial concentration $c_{\imath}$ :

$$
\int_{-\infty}^{\eta /}\left[c_{0}-c(\eta)\right] d \eta=\int_{\pi_{f}}^{\infty}\left[c(\eta)-c_{t}\right] d \eta
$$

The front position of the approximate solution (18) is given by

$$
\eta_{f, a p p}=\eta_{0}+\frac{1}{\beta} \frac{1}{\frac{f_{b}}{c_{b}}-a}\left(\frac{c_{b}-c_{t}}{c_{0}-c_{b}}-\frac{c_{0}-c_{b}}{c_{b}-c_{t}}\right)
$$

and that of the exact solution (20) is given by

$$
\begin{aligned}
\eta_{f, e x}= & \eta_{0}+\frac{1}{c_{0}-c_{\imath}} \frac{2}{\gamma}\left(\frac{\left(c_{b}-c_{t}\right) \sqrt{1+Y}}{Y}+\frac{c_{b}-c_{t}}{Y}\right. \\
& \left.+\frac{\left(c_{b}-c_{t}\right) \sqrt{1+Y}}{X}-\frac{c_{0}-c_{t}}{X}-\frac{c_{0}-c_{b}}{X}\right)
\end{aligned}
$$

In the following, all comparisons of the solutions (18) and (20) are performed with a shock position of $\eta_{f}=0$. This makes it possible to compare the shapes of the front solutions without influences due to a displacement between the fronts.

\section{Discussion}

\subsection{Front Shape}

In order to discuss the shape of the concentration front, it is advantageous to reparameterize (5) or (16), respectively. Both processes, dispersion (characterized by the parameter $P e$ ) and sorption kinetics (characterized by the parameter $L$ ), lead to spreading of the traveling wave front (spreading increases with decreasing values of the parameters $P e$ or $L$ ). The reparameterization

$$
d=\frac{1}{(P e L)^{1 / 2}} \quad p=\left(\frac{P e}{L}\right)^{1 / 2}
$$

with the inversion

$$
P e=\frac{\dot{p}}{d} \quad L=\frac{1}{d p}
$$



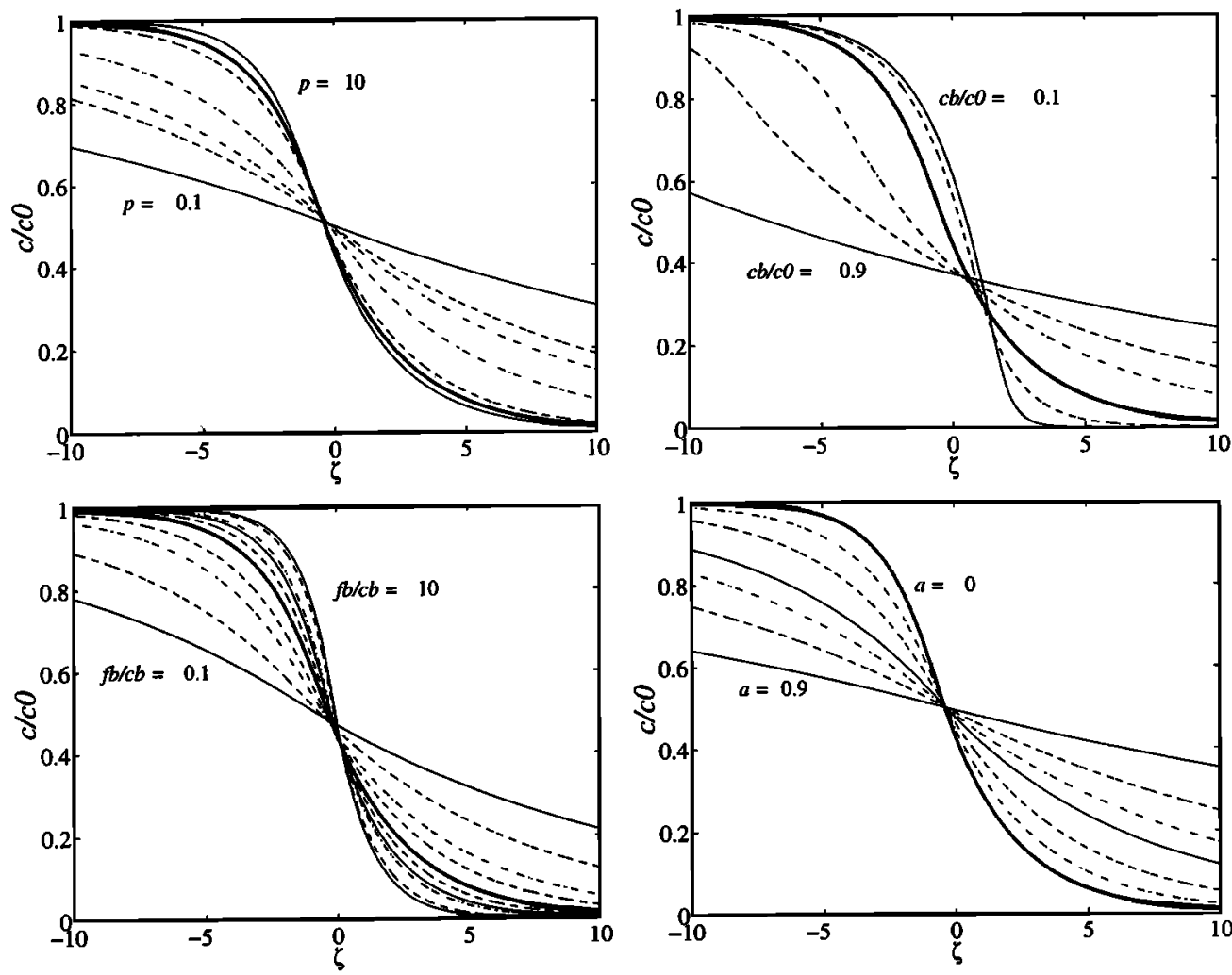

Figure 3. Changes in the front shape for changes in the parameters $p$ (top left: $p=0.1,0.2,0.5,1,3$, and 10 ), $c_{b} / c_{0}$ (top right: $c_{b} / c_{0}=0.1,0.3,0.5,0.7,0.8$, and 0.9 ), $f_{b} / c_{b}$ (bottom left: $f_{b} / c_{b}=0.1,0.2,0.4$, $0.6,1,1.4,2,2.5,6$, and 10 ), and $a$ (bottom right: $a=0,0.2,0.4,0.6,0.7,0.8$, and 0.9 ). The solid line corresponds to the curve for the reference parameter values given by equation (32).

decouples spreading and shape effects by the introduction of a parameter $d$ that quantifies spreading (spreading increases with increasing values of $d$ ) and a parameter $p$ that quantifies the relative importance of spreading due to dispersion $(P e)$ to that due to sorption kinetics $(L)$. For small values of $p$, the dominant cause for spreading is dispersion. For large values of $p$ it is sorption kinetics. The spreading effect can be eliminated from the discussion of front shapes by introducing a new scaled moving space coordinate

$$
\zeta=\frac{\eta}{d}=\eta(P e L)^{1 / 2}
$$

If the reparameterizations (28) and (30) are applied, the lefthand sides of (5) and (16) change to

$$
\alpha \frac{d^{2} c}{d \zeta^{2}}-\left(\frac{1}{p}+p \alpha(1-\alpha)\right) \frac{d c}{d \zeta}=\cdots
$$

Because the wave speed $\alpha$ is independent of $P e$ and $L$ (see (6) and (17)), this expression makes it evident that only the ratio of $P e$ to $L$ described by the parameter $p$ is a relevant parameter for the shape of the traveling wave solutions. Different values of $P e$ and $L$ that lead to the same value of $p=(P e / L)^{1 / 2}$ only stretch or compress the front. This effect is eliminated with the aid of the coordinate $\zeta$.

After applying the transformation described above, setting the initial concentration $c_{\imath}$ to zero, and expressing all concentrations relative to $c_{0}$, the following free parameters remain to determine the front shape: $W, p, c_{b} / c_{0}, f_{b} / c_{b}$, and $a$. The dependence of the front shape on these parameters is discussed with respect to the reference values

$$
W=2 \quad p=1 \quad \frac{c_{b}}{c_{0}}=\frac{1}{2} \quad \frac{f_{b}}{c_{b}}=1 \quad \alpha=0
$$

Because for $a=0$ the dependence of the solutions on $W$ is the same as that on $f_{b} / c_{b}$ (see equations (17)-(23)), changes in the front shape must not be analyzed with respect to the parameter $W$. Figure 3 shows the dependence of the front shape on the four other parameters.

The plots in Figure 3 show that changes in $p, f_{b} / c_{b}$, and $a$ lead to similar changes in the front shape that can hardly be distinguished from stretching or compression. This means that the parameters $p, f_{b} / c_{b}, a$, and $d$ can hardly be identified all together from measured traveling wave front shape data. Decreasing values of $f_{b} / c_{b}$ and increasing values of $a$ lead to increased spreading of the traveling wave fronts. This can qualitatively be understood by the fact that the nonlinearity of the isotherm (angle between the two branches at the break point) decreases with decreasing values of $f_{b} / c_{b}$ and with increasing values of $a$ (see Figure 1). This weakening of the nonlinearity of the isotherm increases spreading because it is the selfsharpening effect of the nonlinear isotherm that working against the spreading effects of dispersion and sorption kinetics leads to the existence of traveling wave solutions. In contrast to the observation that changes in $p, f_{b} / c_{b}$, and $a$ can hardly be distinguished from stretching or compression, the parameter $c_{b} / c_{0}$ significantly influences the front shape. For small values of $c_{b} / c_{0}$ the steepest part of the front is at small concentrations, whereas for large values of $c_{b} / c_{0}$ it is at large concentrations. This can be qualitatively understood by the kinematic effect on the front shape of the corresponding transport prob- 

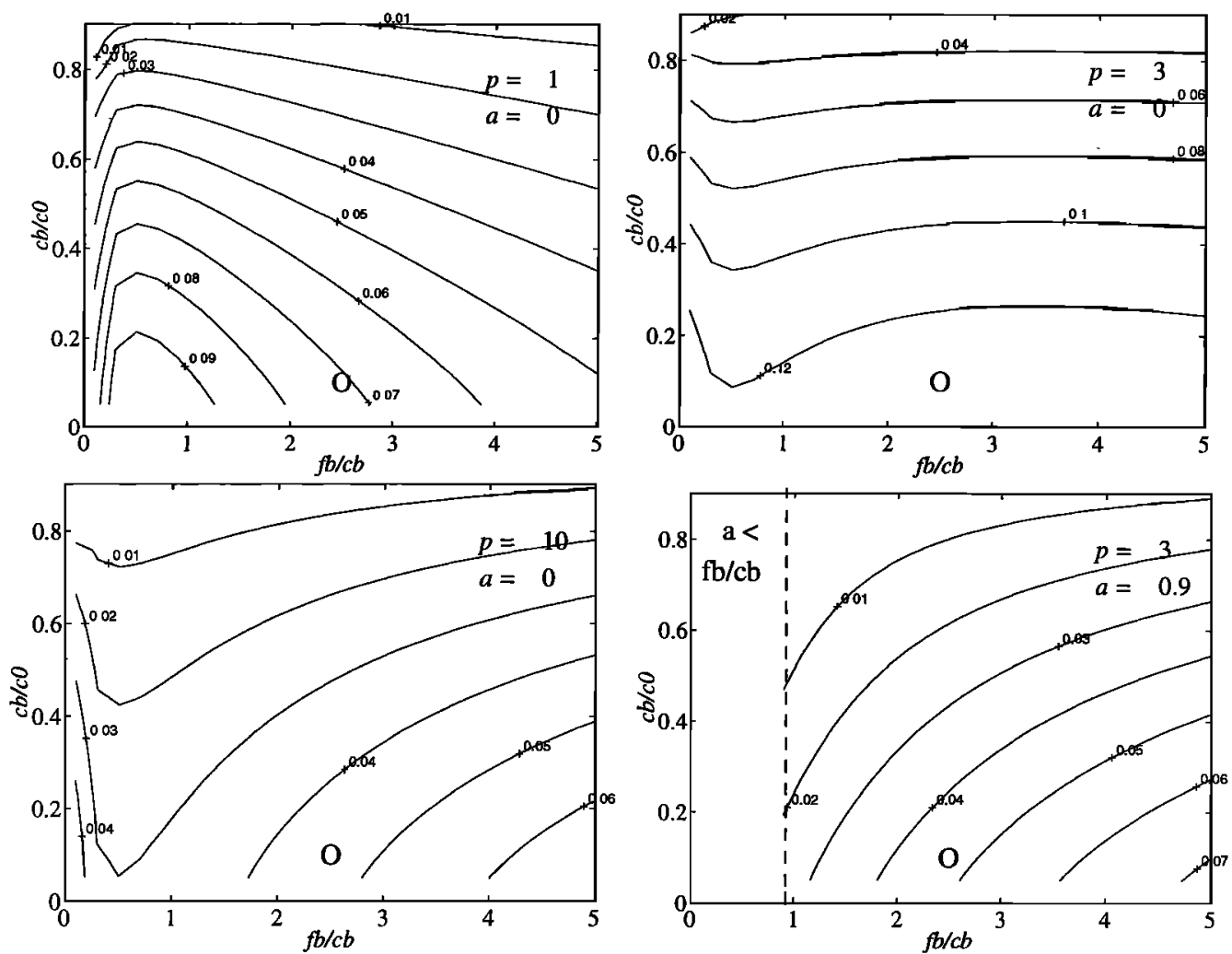

Figure 4. Plots of the dimensionless deviation $\Delta$ between the approximate and exact traveling wave solutions (equation (34)) as a function of $f_{b} / c_{b}$ and $c_{b} / c_{0}$ for $W=2$ and different values of $p$ and $a$. The circle marks the position at which the exact and approximate solutions are compared in Figure 5.

lem without dispersion and with equilibrium sorption. In this case the velocity of a point of the concentration front is given by [Charbeneau, 1981; Helfferich, 1986]

$$
\begin{aligned}
& v_{f}=\frac{\nu}{1+\frac{1}{\theta} \frac{d\left(q_{1}+f\right)}{d c}}=\frac{\nu}{1+\frac{1}{\theta}\left(k_{l}+\frac{f_{b}}{c_{b}}\right)} \quad c \leq c_{b} \\
& v_{f}=\frac{\nu}{1+\frac{1}{\theta} \frac{d\left(q_{1}+f\right)}{d c}}=\frac{\nu}{1+\frac{1}{\theta}\left(k_{l}+a\right)} \quad c>c_{b}
\end{aligned}
$$

This equation shows that the concentration at which the selfsharpening effect is strongest is given by $c_{b}$. For this reason the curves with large values of $c_{b}\left(c_{b} / c_{0}\right.$ close to 1$)$ are steep in the upper part, whereas those with small values of $c_{b}\left(c_{b} / c_{0}\right.$ close to 0$)$ are steep in the lower part of the concentration range. For $c_{i}=0$ the parameter $c_{b} / c_{0}$ characterizes the fraction of the step height $c_{0}$ that is in the linear branch of the isotherm (for $a=0$ the fraction $1-c_{b} / c_{0}$ is in the saturated branch). The results of this section indicate that this parameter can be identified from asymptotic front shape data.

\subsection{Approximation Error}

The deviation of the approximate solution given by (18) from the exact solution given by (20) can be quantified by the maximum of the dimensionless difference

$$
\Delta=\frac{1}{c_{0}} \max _{\eta}\left|c_{a p p}(\eta)-c_{e x}(\eta)\right|
$$

of the two solutions. Figure 4 shows contour lines of this function for different values of the parameters $p$ and $a$ as a function of $f_{b} / c_{b}$ and $c_{b} / c_{0}$. This figure shows that the dimensionless error of the approximate solutions is at most about $12 \%$. It decreases for large and small values of $p$ with a maximum at about $p \approx 3$. This behavior is expected by the form of the left-hand side of (5) and (16) that shows that the second order term can be neglected if only one of the two parameters $P e$ and $L$ is very large. In most cases the error decreases with increasing values of $c_{b} / c_{0}$ and with increasing values of $a$. This shows that a strong nonlinearity of the isotherm increases the error of the approximate solutions (see discussion of the effect of the parameters $c_{b} / c_{0}$ and $a$ on the nonlinearity of the isotherm in the previous subsection).

\subsection{Comparison of Exact and Approximate Solutions}

Figure 5 shows comparisons between the approximate solution (18) and the exact solution (20) for the same sets of parameters $p$ and $a$ as used for plotting the error contour lines in Figure 4 and for $f_{b} / c_{b}=2.5$ and $c_{b} / c_{0}=0.1$ (position marked by a circle in the plots of Figure 4). As expected from the results discussed in the previous subsection the largest error appears for the case with $p=3$ and $a=0$. The general behavior of the deviation is always the same: as a function of the distance $\zeta$ from the front position at $\zeta=0$, the approach of the approximate solution to $c_{0}$ is slower and the approach to $c_{i}$ is faster compared to the exact solution. The reason for this phenomenon can be seen from (5) or (16). If the second-order term is negative (solution approaching $c_{0}$ ), then the magnitude of $d c / d \eta$ decreases. In contrast, $d c / d \eta$ increases if the second-order term is positive (solution approaching $c_{i}=0$ ). The comparison of exact and approximate solutions shows that the error caused by neglecting the second-order term is usually small and hardly measurable. Rather extreme conditions (e.g., 

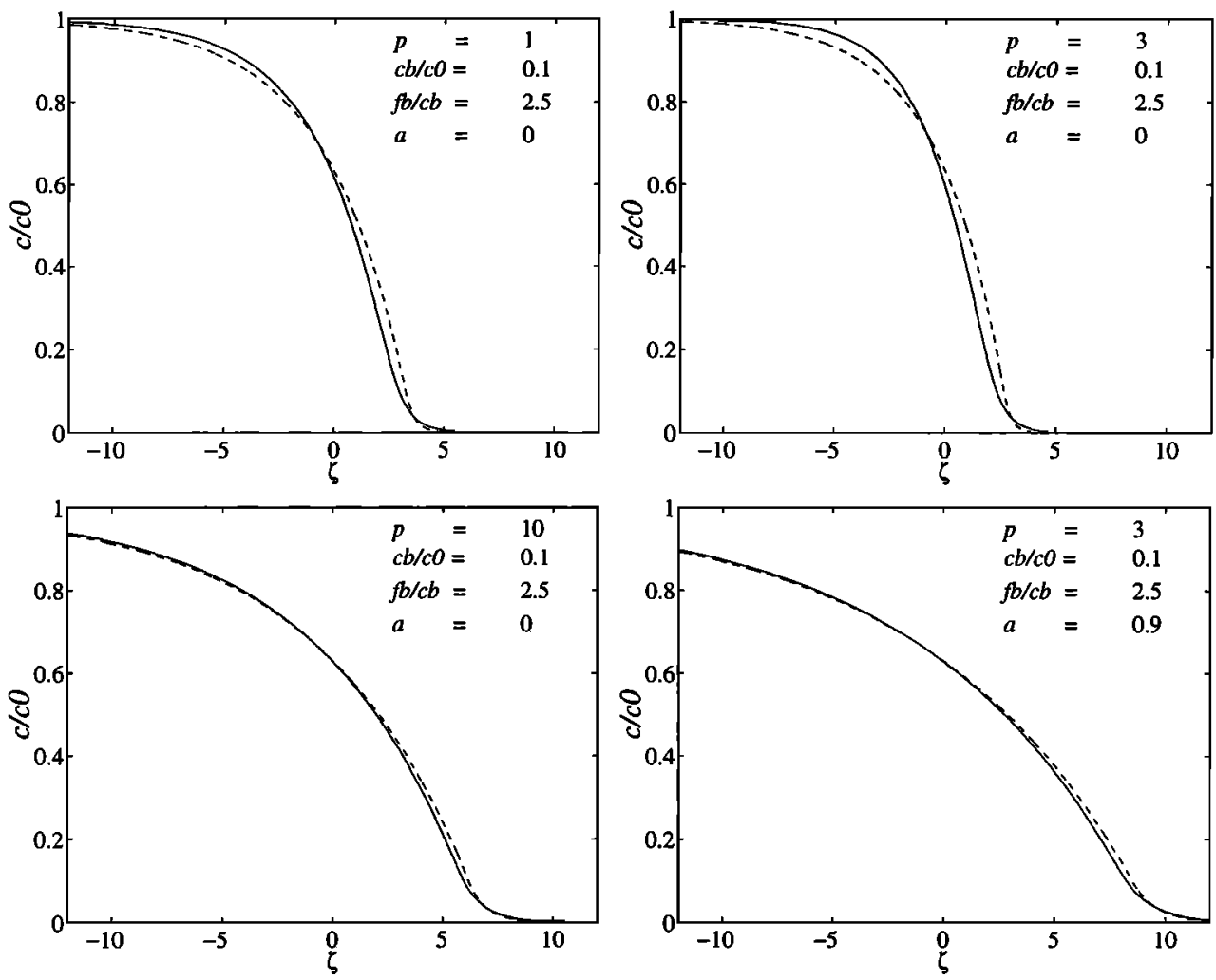

Figure 5. Comparisons of the exact solution (solid line) with the approximate solution (dashed line) for the same sets of parameters $p$ and $a$ as used for plotting the error contour lines in Figure 4 and for $f_{b} / c_{b}=2.5$ and $c_{b} / c_{0}=0.1$ (position marked by a circle in the plots of Figure 4).

$\left.c_{b} / c_{0}=0.1\right)$ are necessary in order to obtain a significant deviation in the front shape. This confirms that the secondorder term can usually be neglected.

\subsection{Consequences for Process Identification}

Only the left-hand side of the traveling wave equation (5) depends on the parameters $P e$ and $L$. The form of this dependence given by

$$
\left(\frac{\alpha}{P e L}\right) \frac{d^{2} c}{d \eta^{2}}-\left(\frac{1}{P e}+\frac{\alpha(1-\alpha)}{L}\right) \frac{d c}{d \eta}=\cdots
$$

shows that the second-order term can be neglected if one of the parameters $P e$ or $L$ is much larger than the other. In this case the effects of dispersion (described by $P e$ ) and of sorption kinetics (described by $L$ ) cannot be distinguished from each other, because both parameters only affect the coefficient of the first order term. More precisely, the two choices of parameters given by

$$
\begin{gathered}
P e=P e_{0} \quad L=\infty \\
P e=\infty \quad L=L_{0}=P e_{0} \alpha(1-\alpha)
\end{gathered}
$$

lead to exactly the same traveling wave differential equation and therefore to the same solutions. This means that an effect of the second-order term is the only possibility to distinguish the effects of dispersion from those of sorption kinetics from asymptotic front data if both processes are present simultaneously. As was shown in the preceeding section, the effect of the second-order term is usually very small, so that a separation of these two spreading processes from asymptotic front data is extremely difficult.
In this section, with the aid of numerical preasymptotic solutions to (4) for the isotherm given by (13) and for both choices of parameters given above in (36a) and (36b), it is shown that these two processes lead to slightly different behavior for preasymptotic conditions and that they can best be distinguished with the aid of experiments in which the flow is interrupted while the concentration increases or decreases significantly at the column outlet [Brusseau et al., 1989]. The numerical calculations for this section were performed with an extension of the program AQUASIM [Reichert, 1994, 1995] to soil columns. The propagation of a front resulting from a step increase of the input flux was calculated for a soil column long enough that the exit boundary condition was not relevant. Figure 6 shows comparisons of the calculated spatial profiles for two different points in time after the increase of the input flux and concentration time series at a given location for interrupted flow experiments. Note that the breakthrough of the main front is faster for the choice given by (36b) because the slowness of the adsorption process diminishes retardation. The shape of the profiles is only slightly different for the case with dispersion (36a) as compared to the case with sorption kinetics (36b) also in the preasymptotic range shown by the first profiles $(T=1)$. The second numerically calculated profiles shown in Figure $6(T=6$ or $T=10$, respectively) are already indistinguishable from the analytical solutions calculated with the aid of (20).

\section{Conclusions}

Analytical traveling wave solutions for a substance transported dispersively in a porous medium in the presence of 

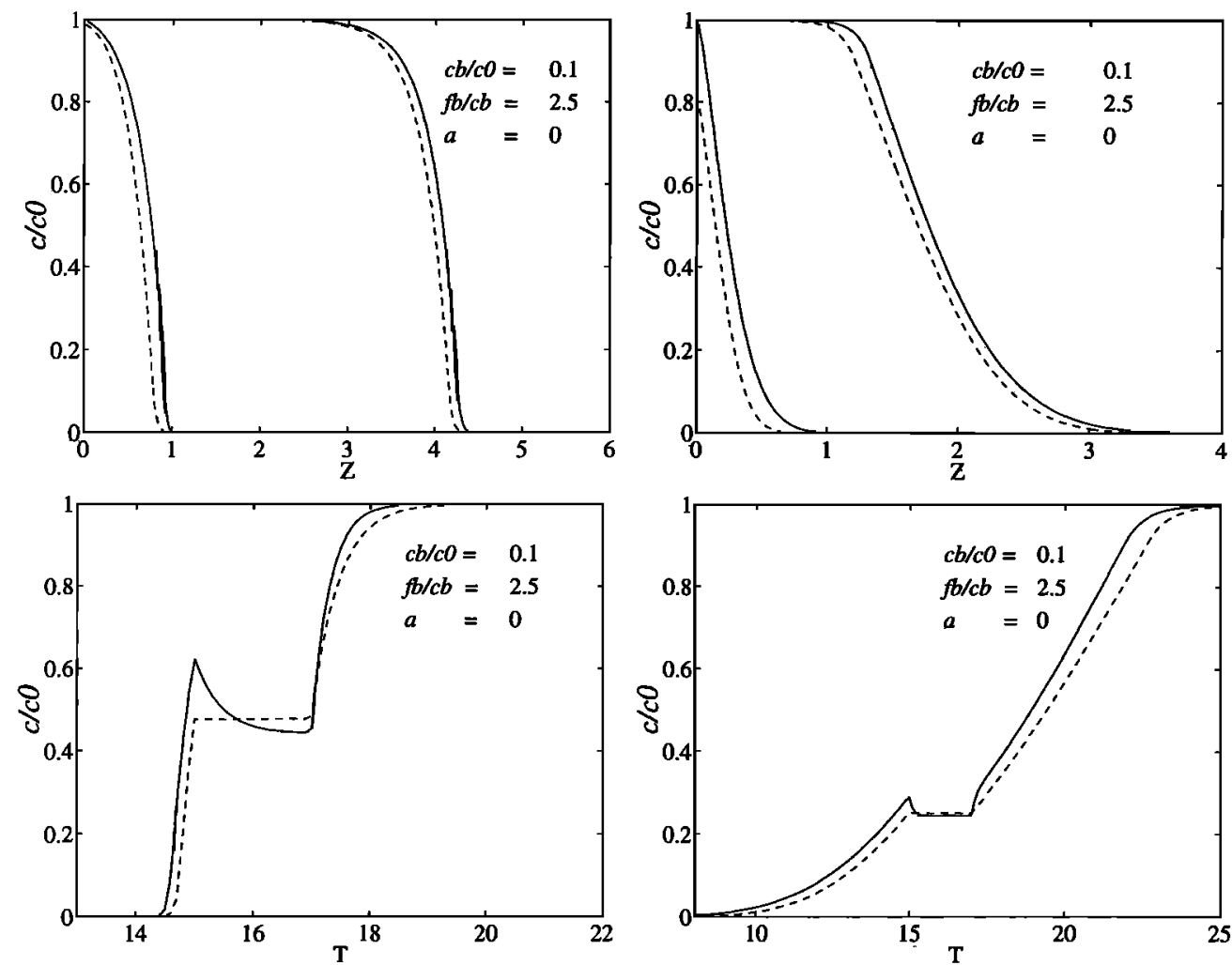

Figure 6. Comparison of preasymptotic solutions ( $T=1$ and $T=6$, left, and $T=1$ and $T=10$, right) that lead to the same asymptotic traveling waves (top) and concentration time series for interrupted flow experiments at $Z=10$ (left) and at $Z=3$ (right) (bottom). In all plots a step increase in the flux at $Z=$ 0 occurs at $T=0$ and solid lines represent solutions with dispersion only (equation (36a)), and dashed lines represent solutions with sorption kinetics only (equation (36b)). For the plots in the bottom row, flow interruption is from $T=15$ to $T=17$.

linear equilibrium and nonlinear nonequilibrium sorption have been derived for a simple piecewise linear sorption isotherm. These analytical solutions make it possible to demonstrate the effect of neglecting the second-order term of the traveling wave equation (as it is usually done), to discuss the sensitivity of the solutions to changes in the values of model parameters and to assess the identifiability of model parameters from asymptotic front shape data.

It was shown that the solution to the approximate traveling wave equation without the second-order term led to an error up to $12 \%$ of the step concentration. The magnitude of the error is especially large if a large fraction of the concentration step is in the range that leads to a saturation of the sorption process and if dispersion and sorption kinetics have both a similarly large spreading effect on the concentration front. These rather extreme conditions, together with the maximum size of the error that may be in the range of the experimental uncertainty, justify the omission of the second-order term for most applications.

A sensitivity analysis of the front shape to changes in model parameters showed that most parameters have a similar effect on the front shape. Only one parameter, the fraction $c_{b} / c_{0}$ of the step size that leads to linear sorption, significantly effects the front shape in a different way.

The results of the sensitivity analysis given above indicate a bad practical identifiability [Godfrey and DiStefano, 1985, 1987] of the model parameters from measured front shape data. It is easy to see that in the cases in which the spreading effect on the concentration front is caused either by dispersion alone or by sorption kinetics alone it is even theoretically nonidentifiable from asymptotic front shape data which of the two processes is in effect. For this case the two processes can only be distinguished with the aid of data from the preasymptotic range. With the aid of numerical solutions to the partial differential equation describing transport and kinetic sorption, it was shown that it is also difficult to separate these processes with such data for steady state flow. The identifiability of the processes increases significantly if interrupted flow experiments can be performed.

The results of our paper confirm that the second-order term of the traveling wave equation can usually be neglected (as is done for most analytical traveling wave solutions). Hints are given to decide in which cases the term may be important. The properties of the analytical solutions (especially the dependence of the front shape on the model parameters) indicate a bad identifiability of the model parameters from asymptotic front shape data. This means that in order to identify processes and parameters, additional data from the preasymptotic range should be available. If experiments can be performed, flow interruption experiments can much clearer distinguish sorption kinetics from dispersion [Brusseau et al., 1989]. Data from the asymptotic range can then be useful as an additional but not the sole source of information for process and parameter identification. 
Our method of using a piecewise linear sorption isotherm in order to derive analytical traveling wave solutions can be generalized to isotherms that by using more linear pieces, lead to a better approximation of actual isotherm data. However, we suppose that the major conclusions of this paper are not sensitive to the exact form of the isotherm.

Acknowledgments. We thank Christa Bürgisser, Emil Franov, Peter Holzer, Wolfgang Kinzelbach, Andrea Rüede, Rien van Genuchten, and Oskar Wanner for helpful discussions; Gérard Mohler, Gabriel Piepke, and Raoul Schaffner for their technical support; and Annette Johnson and two anonymous reviewers for their comments on the manuscript.

\section{References}

Bolt, G., Movement of solutes in soil: Principles of adsorption/ exchange chromatography, in Soil Chemistry B, Physico-Chemical Models, edited by G. Bolt, pp. 285-348, Elsevier, New York, 1982.

Bosma, W., and S. van der Zee, Analytical approximation for nonlinear adsorbing solute transport and first-order degradation, Transp. Porous Media, 11, 33-43, 1993.

Brusseau, M., P. Rao, R. Jessup, and J. Davidson, Flow interruption: A method for investigating sorption nonequilibrium, $J$. Contam. Hydrol., 4, 233-240, 1989.

Charbeneau, R., Groundwater contaminant transport with adsorption and ion exchange chemistry: Method of characteristics for the case without dispersion, Water Resour. Res., 17(3), 705-713, 1981.

Godfrey, K., and J. DiStefano, Identifiability of model parameters, in Identification and System Parameter Estimation 1985-Proceedings of the Seventh IFAC/IFORS Symposium, vol. 1, edited by $\mathrm{H}$. Barker and P. Young, Pergamon, pp. 89-114, Tarrytown, N. Y., 1985.

Godfrey, K., and J. DiStefano, Identifiability of model parameters, in Identifiability of Parametric Models, edited by E. Walter, Pergamon, pp. 1-20, Tarrytown, N. Y., 1987.

Helfferich, F., Theory of multicomponent chromatography: A state of the art report, J. Chromatog., 373, 45-60, 1986.

Helfferich, F., and G. Klein, Multicomponent Chromatography, Marcel Dekker, New York, 1970.

Lighthill, J., Waves in Fluıds, Cambridge Univ. Press, New York, 1978.

Reichert, P., AQUASIM-A tool for simulation and data analysis of aquatic systems, Water Sci. Technol., 30(2), 21-30, 1994.
Reichert, P., Design techniques of a computer program for the identification of processes and the simulation of water quality in aquatic systems, Environ. Software, 10(3), 199-210, 1995.

Rhee, H.-K, and N. Amundson, A study of the shock layer in nonequilibrium exchange systems, Chem. Eng. Sci., 27, 199-211, 1972.

Rhee, H.-K., and N. Amundson, Shock layer in two solute chromatography: Effect of axial dispersion and mass transfer, Chem. Eng. Sci., 29, 2049-2060, 1974.

Rhee, H.-K., B. Bodin, and N. Amundson, A study of the shock layer in equilibrium exchange systems, Chem. Eng. Sci., 26, 1571-1580, 1971.

Rhee, H.-K., R. Aris, and N. Amundson, First-Order Partial Differential Equations, vol. II, Theory and Application of Hyperbolic Systems of Quasilinear Equations, Prentice Hall, Englewood Cliffs, N. J., 1989.

Schweich, D., M. Sardin, and M. Jauzein, Properties of concentration waves in presence of nonlinear sorption, precipitation/dissolution, and homogeneous reactions, 1, Fundamentals, Water Resour. Res., 29(3), 723-733, 1993 a.

Schweich, D., M. Sardin, and M. Jauzein, Properties of concentration waves in presence of nonlinear sorption, precipitation/dissolution, and homogeneous reactions, 2, Illustrative examples, Water Resour. Res., 29(3), 735-741, 1993b.

Smoller, J., Shock Waves and Reaction-Diffusion-Equations, SpringerVerlag, New York, 1983.

van der Zee, S., Analytical traveling wave solutions for transport with nonlinear and nonequilibrium adsorption, Water Resour. Res., 26(10), 2563-2578, 1990.

van Duijn, C., and P. Knabner, Travelling waves in the transport of reactive solutes through porous media: Adsorption and binary ion exchange, 1, Transp. Porous Media, 8, 167-194, 1992a.

van Duijn, C., and P. Knabner, Travelling waves in the transport of reactive solutes through porous media: Adsorption and binary ion exchange, 2, Transp. Porous Media, 8, 199-225, 1992b.

Whitham, G. B., Linear and Nonlinear Waves, John Wiley, New York, 1974.

C. Hinz, Institute of Terrestrial Ecology, Swiss Federal Institute of Technology, CH-8952 Schlieren, Switzerland.

P. Reichert and W. Simon, Swiss Federal Institute for Environmental Science and Technology, Überlandstrasse 133, CH-8600 Dübendorf, Switzerland. (e-mail: simon@eawag.ch)

(Received June 12, 1996; revised November 1, 1996; accepted January 29, 1997.) 